\title{
Singularity Subtraction in a Multidimensional Fredholm Integral Equation of the Second Kind with a Singular Kernel
}

\author{
Josef $\operatorname{Rak}^{1, a)}$ \\ ${ }^{1}$ University of Pardubice, Faculty of Electrical Engineering and Informatics, Studentska 9553210 Pardubice, Czech \\ Republic. Some results were obtained by author's doctoral study in Charles University in Prague, Faculty of \\ Mathematics and Physics, Department of Numerical Mathematics \\ ${ }^{a)}$ Corresponding author: josef.rak@upce.cz \\ URL: http://fei.upce.cz
}

\begin{abstract}
A numerical solution of the Fredholm integral equations can be obtained by many methods. Most of them lead to a solution of a system of linear equations with fully populated matrices. In the case of collocation or product integration methods, each element of the matrix is an integral, which needs to be calculated. It causes high computing time in multidimensional problems. Computing time can be reduced by the Nyström method. It is based on substitution of the integral by a numerical integration rule. It has the advantage that only diagonal elements of the matrix are integrals. When the kernel function is singular, a singularity subtraction is needed. However it can not be used for every kernel function and every integration rule. The main point of this paper is the convergence conditions of the Nyström method as applied to a special multidimensional integral equation. The paper includes an illustrative example.
\end{abstract}

\section{INTRODUCTION}

Let us have an integral equation of the second kind of the form

$$
\lambda y(x)-\int_{D} k(x, t) y(t) d t=f(x), x \in D, \lambda \neq 0,
$$

where $D \subset R^{m}(m \geq 1)$ is a closed, bounded and connected set. Assume that the kernel function $k(x, t)$ is singular when $x=t$. Such a kernel function includes $k(x, t)=r^{-1}(x, t)$, where $r(x, t)$ is the Euclidean distance. The existence and uniqueness of the solution is done by operator calculus and is well described by many books (for example [1]). Equation (1) is rewritten into an operator form $(\lambda I-\mathcal{K}) y=f$, where the integral operator $\mathcal{K}$ is defined by

$$
\mathcal{K} y(x)=\int_{D} k(x, t) y(t) d t .
$$

Assume that the operator $\mathcal{K}$ is a compact operator on $C(D)$. Compactness of operator $\mathcal{K}$ can be proved for $k(x, t)=$ $r^{-1}(x, t)$ if $m=1,2,3$. From the Fredholm alternative theorem (1) has a unique solution if $\lambda \neq 0$ is not an eigenvalue of operator $\mathcal{K}$.

\section{NYSTRÖM METHOD}

One numerical method for solution of (1) is the Nyström method. It is based on the substitution of the integral by a numerical integration rule. The kernel function $k(x, t)$ is singular when $x=t$. So the numerical integration rule can not be used directly. First let us rewrite (1) into the form as in [4]

$$
\left[\lambda-\int_{D} k(x, t) d t\right] y(x)-\int_{D} k(x, t)[y(t)-y(x)] d t=f(x) .
$$


By replacement of the kernel function $k(x, t)$ with its bounded approximation $k_{n}(x, t)$ in the second integral on the left hand side of (3), substitution of the second integral in (3) with a numerical integration rule, and running $x$ through the numerical integration rule node points, we get a system of linear equations for the numerical solution $y_{n}\left(x_{i}\right)$ at the node points $x_{i}$

$$
\left[\lambda+\sum_{j=1, j \neq i}^{n} \omega_{j} k_{n}\left(x_{i}, x_{j}\right)-\int_{D} k\left(x_{i}, t\right) d t\right] y_{n}\left(x_{i}\right)-\sum_{j=1, j \neq i}^{n} \omega_{j} k_{n}\left(x_{i}, x_{j}\right) y_{n}\left(x_{j}\right)=f\left(x_{i}\right),
$$

where $\omega_{i}$ are the integration rule's weights. The numerical solution at other points $x \in D$ is obtained by the interpolation formula

$$
y_{n}(x)=\frac{f(x)+\sum_{j=1}^{n} \omega_{j} k_{n}\left(x, x_{j}\right) y_{n}\left(x_{j}\right)}{\lambda+\sum_{j=1}^{n} \omega_{j} k_{n}\left(x, x_{j}\right)-\int_{D} k(x, t) d t} .
$$

The integral in (4) and (5) can be calculated analytically or by some special numerical integration rule. Construction of the bounded approximation $k_{n}(x, t)$ is also important. Convergence of this method requires special conditions for the kernel function $k(x, t)$ and the numerical integration rule. In a one-dimensional case the construction of $k_{n}(x, t)$ and convergence conditions were done by Anselone's theory of collectively compact operators (see [2]) and can be found in [3]. Generalization to multidimensional problems was done by the author in his doctoral thesis [6]. The most important results are summarized in the following two sections.

\section{KERNEL FUNCTION CONDITIONS}

In this section let us formulate conditions for the kernel function. Assume that there exists a function $h \in C(D \times D)$ and the positive non-increasing function $g \in C(0, \infty)$, which satisfies

$$
\lim _{t \rightarrow 0+} g(t)=\infty
$$

such that the kernel function is of the form

$$
k(x, t)=g(r(x, t)) h(x, t)
$$

Assume that there exists a constant $c_{D}<\infty$ such that

$$
\max _{x \in D} \int_{\left\{t, r(x, t)<R_{D}\right\}} g(r(x, t)) d t \leq c_{D}, \text { where } R_{D}=\max _{x, t \in D} r(x, t) .
$$

and

$$
\lim _{\nu \rightarrow 0} \max _{x \in D} \int_{\{t, r(x, t)<v\}} g(r(x, t)) d t=0 .
$$

Then kernel function approximation is defined by

$$
k_{n}(x, t)=g_{\mu_{n}}(r(x, t)) h(x, t), \text { where } g_{\mu_{n}}(u)=\left\{\begin{array}{l}
g(u), \text { if } u \geq \mu_{n} \\
g\left(\mu_{n}\right), \text { if } u<\mu_{n},
\end{array}\right.
$$

where $\mu_{n}$ is a positive decreasing sequence which has

$$
\lim _{n \rightarrow \infty} \mu_{n}=0 .
$$

Note that $g_{\mu_{n}} \in C[0, \infty)$ is also a positive and non-increasing function for all $n$.

\section{NUMERICAL INTEGRATION RULE CONDITION}

Now let's formulate important conditions for the numerical integration rule. Assume that it is convergent for all continuous functions and has positive weights. Let us define

$$
\bar{\omega}_{n}=\max _{j=1, \ldots, n} \omega_{j}
$$


For the sequence $\mu_{n}$ assume that

$$
\mu_{n}^{m} \geq \rho^{m} \bar{\omega}_{n}
$$

where $0<\rho<\infty$. Also assume that there exists $\bar{\mu}<\infty$ such that

$$
g\left(\mu_{n}\right) \bar{\omega}_{n} \leq \bar{\mu} \text { for all } n \text {. }
$$

Finally assume that there exist constants $c<\infty$ and $0<\xi<\infty$ such that for all positive, non-increasing function $z \in C[0, \infty)$ and $x \in D$, it holds

$$
\sum_{j, r\left(x, x_{j}\right) \leq \xi} \omega_{j} z\left(r\left(x, x_{j}\right)\right) \leq c\left[z(0) \bar{\omega}_{n}+\int_{\{t, r(x, t) \leq \xi\}} z(r(x, t)) d t\right] .
$$

Now let us formulate two theorems for multidimensional integration rules that satisfy condition (15).

Theorem 1 Let the domain $D=\left\{\left(x_{1}, x_{2}\right), a \leq x_{1} \leq b, c \leq x_{2} \leq d\right\}$ be a rectangle partitioned by $n_{1} n_{2}$ subrectangles $\left(n_{1}\right.$ in $x_{1}$ direction and $n_{2}$ in $x_{2}$ direction). Let $h_{1}=(b-a) n_{1}^{-1}$ and $h_{2}=(d-c) n_{2}^{-1}$. Let $z \in C[0, \infty)$ be a positive and non-increasing function. Let the numerical integration rule be the compound mid-rectangular rule and assume that there exists $1 \leq \alpha<\infty$ such that $h_{1}=\alpha h_{2}$. Then there exist constants $c$ and $\xi$ such that (15) is satisfied.

Theorem 2 Let the domain $D=\left\{\left(x_{1}, x_{2}, x_{3}\right), a \leq x_{1} \leq b, c \leq x_{2} \leq d, e \leq x_{3} \leq f\right\}$ be a cuboid partitioned by $n_{1} n_{2} n_{3}$ sub-cuboids ( $n_{1}$ in $x_{1}$ direction, $n_{2}$ in $x_{2}$ direction and $n_{3}$ in $x_{3}$ direction). Let $h_{1}=(b-a) n_{1}^{-1}, h_{2}=(d-c) n_{2}^{-1}$ and $h_{3}=(f-e) n_{3}^{-1}$. Let $z \in C[0, \infty)$ be a positive and non-increasing function. Let the numerical integration rule be the compound mid-cuboid rule and assume there exists $1 \leq \beta \leq \alpha<\infty$ such that $h_{1}=\alpha h_{3}$ and $h_{2}=\beta h_{3}$. Then there exist constants $c$ and $\xi$ such that (15) is satisfied.

Note that the assumptions of type $h_{1}=\alpha h_{2}$ can be changed to $h_{2}=\alpha h_{1}$ in theorem 1 . Similar assumption in theorem 2 can be also changed. These assumption are only technical.

\section{INTEGRAL EQUATION FOR INDUCTION HEATING}

Let use the theory above to compute Joule losses in the cuboid metal body $\Omega_{1}=\left\{\left(x_{1}, x_{2}, x_{3}\right), a \leq x_{1} \leq b, c \leq x_{2} \leq\right.$ $\left.d, e \leq x_{3} \leq f\right\}$, which is heated by a coil $\Omega_{2}$. The formula for Joule losses (see [5]) is

$$
\omega_{J}(x)=\frac{1}{\gamma} J_{e}(x) \overline{J_{e}(x)}
$$

where

$J_{e}(x)=\sqrt{\left[\operatorname{ReJ}_{e d d y, x_{1}}(x)\right]^{2}+\left[\operatorname{ReJ}_{e d d y, x_{2}}(x)\right]^{2}+\left[\operatorname{Re} J_{e d d y, x_{3}}(x)\right]^{2}}+\iota \sqrt{\left[\operatorname{Im} J_{e d d y, x_{1}}(x)\right]^{2}+\left[\operatorname{Im} J_{e d d y, x_{2}}(x)\right]^{2}+\left[\operatorname{Im} J_{e d d y, x_{3}}(x)\right]^{2}}$,

$\overline{J_{e}(x)}$ is a complex conjugate to $J_{e}(x), J_{e d d y}=\left(J_{e d d y, x_{1}}, J_{e d d y, x_{2}}, J_{e d d y, x_{3}}\right)$ is a phasor of the eddy currents of density. The eddy currents of density phasor is obtained by

$$
\iota J_{e d d y}(x)-\kappa(x) \int_{\Omega_{1}} \frac{J_{e d d y}(t)}{r(x, t)} d t_{1} d t_{2} d t_{3}=\kappa(x) I_{\text {ext }} \int_{\Omega_{2}} \frac{d l(s)}{r(x, s)}, \text { where } \kappa(x)=\frac{\omega \gamma(T(x)) \mu_{0}}{4 \pi},
$$

where $x, t$ are points in the heated body, $s$ is a point at the inductor, $r(x, t)$ is the Euclidean distance, $\iota$ is a complex unit, $\mu_{0}$ is the permeability of vacuum, $d l(s)$ is a length element of the inductor, $\omega$ denotes angular frequency of the field current, $\gamma$ denotes the temperature dependent electrical conductivity of the metal, $I_{\text {ext }}$ is field current and $T(x)$ is the temperature in the body at point $x$. Equation (17) is of the form (1) with $\lambda=1, k(x, t)=-\iota \kappa(x) r(x, t)^{-1}$ and

$$
f(x)=-\iota \kappa(x) I_{e x t} \int_{\Omega_{2}} \frac{d l(s)}{r(x, s)} .
$$

Using sphere coordinates, the corresponding integral operator can be proved to be a compact operator on $C(D)$. With $\frac{1}{\kappa}$-weighted inner product, the integral operator is antisymmetric and $\lambda=1$ is not its eigenvalue. So equation (17) has 
a unique solution. Let the numerical integration rule be a compound mid-cuboid rule with $n$ sub-cuboids. The kernel function $k(x, t)$ is of the form (7) with $h(x, t)=-\iota k(x)$ and $g(u)=u^{-1}$. By (10) we have the bounded approximation

$$
k_{n}(x, t)=\frac{-\iota \kappa(x)}{r_{n}(x, t)}, \text { where } r_{n}(x, t)=\left\{\begin{array}{l}
r(x, t) \text { if } r(x, t) \geq \mu_{n} \\
\mu_{n} \text { if } r(x, t)<\mu_{n}
\end{array} \text { and } \mu_{n}=\sqrt[3]{\frac{(b-a)(d-c)(f-e)}{n}} .\right.
$$

Such a kernel function and its approximation can be proved to satisfy conditions (6)-(11). The compound midcuboid rule and kernel function can be proved to satisfy conditions (13), (14). Finally by theorem 2 the compound mid-cuboid rule satisfies (15). For detail see [6]. So, conditions for a convergence of the described method are satisfied.

\section{EXAMPLE}

A brass cuboid body with the sizes $0.15 \times 0.01 \times 0.01 \mathrm{~m}$ is heated with a stationary inductor starting at a room temperature of $20^{\circ} \mathrm{C}$. The inductor is a coil which turns around the heated body in the $x_{1}$-direction in 6 loops. The radius of the coil is $0.015 \mathrm{~m}$, exciting current is $500 \mathrm{~A}$ and frequency is $150 \mathrm{kHz}$. The length of the coil is $0.15 \mathrm{~m}$. The cuboid is partitioned by 75 elements in $x_{1}$ direction, 5 elements in $x_{2}$ and $x_{3}$ direction. The middle picture in figure 1 shows the Joule losses at the $x_{1}$ axes with $x_{2}=-0.004$. The third picture shows Joule losses at the $x_{1}$ axes with $x_{2}=0$. The blue color matches $x_{3}=-0.004$, the red color matches $x_{3}=0$ and the black color matches $x_{3}=0.004$.
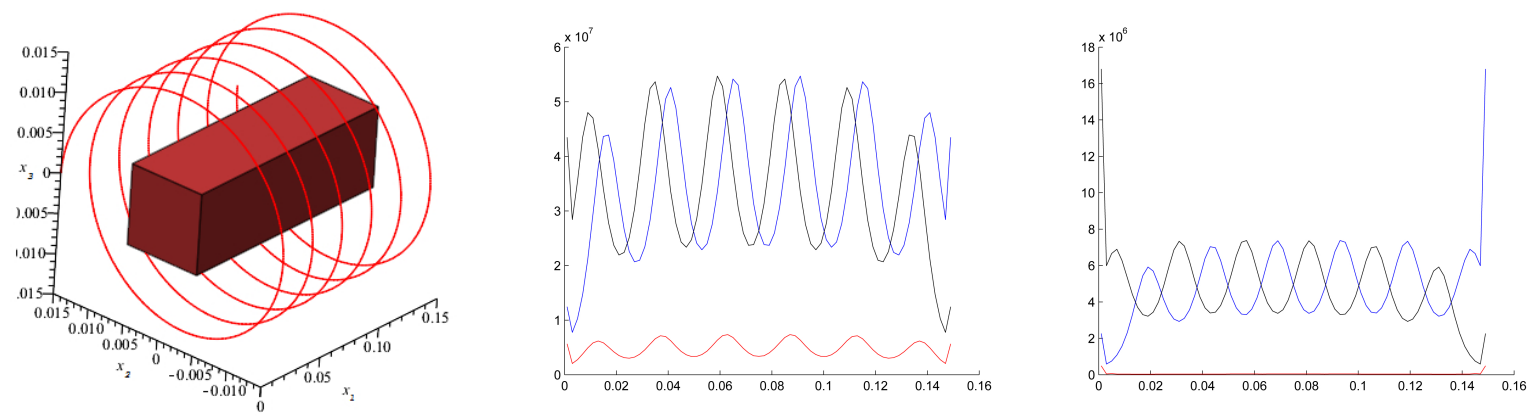

FIGURE 1. Induction heating example - Joule losses

\section{CONCLUSION}

The disadvantage of the Nyström method with singularity subtraction is error analysis. It has special conditions for the kernel function and the integration rule. However it has lower computing time. If we compare computing time on Windows 7 operating system with Intel Core i5 and 4GB RAM we get approximately 2 minutes for the Nyström method and approximately 12 minutes for piecewise constant collocation. This is the goal of described method .

\section{REFERENCES}

[1] K. E. Atkinson: The Numerical Solution of Integral Equations of the Second Kind (Cambridge University Press, 1997)

[2] P. M. Anselone: Collectively compact operator approximation theory and applications to integral equations (Prentice-Hall, Inc., Englewood Cliffs, N.J. 1971)

[3] P. M. Anselone: Singularity Subtraction In The Numerical Solution Of Integral Equations, J. Austral. Math. Soc. (Series B) 22 (1981), p. 408-418.

[4] L. V. Kantorovich, V. I. Krylov: Approximate Methods of Higher Analysis, (Interscience, New York, 1958)

[5] P. Solin, I. Dolezel, M. Skopek, B. Ulrych, SPETO 2001 International Conference, 143-146 (2001)

[6] J. Rak, Numerical Solution of a Fredholm Integral Equation of the Second Kind Related to Induction Heating, Praha 2012. Doctoral thesis. Charles University Prague, Faculty of Mathematics and Physics, 27.9.2012 